\title{
Stability analysis of networked control systems subject to packet- dropouts and finite-level quantization
}

\section{$\operatorname{AUTHOR}(\mathrm{S})$ :}

Ishido, Yumiko; Takaba, Kiyotsugu; Quevedo, Daniel E.

\section{CITATION:}

Ishido, Yumiko ...[et al]. Stability analysis of networked control systems subject to packetdropouts and finite-level quantization. Systems \& Control Letters 2011, 60(5): 325-332

\section{ISSUE DATE:}

2011-05

URL:

http://hdl.handle.net/2433/141828

\section{RIGHT:}

(c) 2011 Elsevier B.V.; This is not the published version. Please cite only the published version.; この論文は出版社版でありません。引用の際に は出版社版をご確認ご利用ください。 


\title{
Stability Analysis of Networked Control Systems Subject to Packet-dropouts and Finite Level Quantization
}

\author{
Yumiko Ishido $^{\mathrm{a}, *}$, Kiyotsugu Takaba ${ }^{\mathrm{a}}$, Daniel E. Quevedo ${ }^{\mathrm{b}}$ \\ ${ }^{a}$ Department of Applied Mathematics and Physics, Graduate School of Informatics, \\ Kyoto University, Kyoto 606-8501, Japan, Tel:+81-75-753-5512, Fax:+81-75-753-5507 \\ ${ }^{b}$ School of Electrical Engineering 83 Computer Science, The University of Newcastle, \\ Callaghan, NSW 2308, Australia
}

\begin{abstract}
This paper is concerned with stability analysis of a networked control system, wherein communication from the controller to the plant input is through a digital channel subject to packet-dropouts and finite level quantization. No acknowledgments of receipt are available to the controller. To alleviate the effect of packet-dropouts, the controller transmits tentative plant input sequences. Within this setup, we derive a sufficient condition for small $\ell^{\infty}$ signal $\ell^{\infty}$ stability of the networked control system. This condition requires the maximum number of consecutive packet-dropouts to be bounded. We also elucidate the trade-off which exists between the disturbance attenuation and the step size of the quantizer and the maximum number of consecutive packet-dropouts.
\end{abstract}

Keywords:

Networked control systems, finite level quantization, packet-dropouts, stability analysis, buffering

\footnotetext{
*Corresponding author.

Email addresses: ishido@amp.i.kyoto-u.ac.jp (Yumiko Ishido), takaba@amp.i.kyoto-u.ac.jp (Kiyotsugu Takaba), dquevedo@ieee.org (Daniel E. Quevedo)
} 


\section{Introduction}

Networked control systems (NCSs) are composed of sets of plants and controllers which are connected with each other through shared communication networks. In NCSs, the control performance of the entire systems is affected by network properties such as graph topology [1], data rate [2, 3], packet-dropouts $[4,5,6]$ and delays $[7,8]$.

Recently, some works have discussed the stability of the single-loop NCSs affected by both packet-dropouts and quantization in stochastic settings [9],[10],[12]. Tsumura, Ishii and Hoshina [9] and Niu et al. [10] independently studied the mean-square stability of the NCS in the case of a logarithmic quantizer with an infinite number of quantization levels, and clarified the trade-off between the coarseness of the quantizer and the stability of the NCS. For finite-level quantizers, Matveev and Savkin [12] obtained a negative result, namely, they showed that an unstable linear plant subject to arbitrarily and uniformly small external disturbances can never be almost surely stabilized in the presence of packet-dropouts.

In contrast with the above previous works, this paper will take an alternative look at the stability analysis of NCSs with both packet-dropouts and quantization To be more specific, we will consider a deterministic setting, and study stability of NCSs where

- the number of connective packet-dropouts is bounded, and

- the quantizer has only a finite number of quantization levels.

Within the setup considered, a buffering mechanism $[15,16,17,18]$ is incorporated into the NCS to cope with the adverse effect of packet-dropouts: the controller transmits packets consisting of predictions of quantized control inputs, and the buffer at the actuator side determines the current control input based on the buffered packet and transmission outcomes.

For the analysis of the NCS with both finite level quantization and packetdropouts, we will employ the notion of small $\ell^{p}$ signal $\ell^{p}$ stability, which was recently proposed by Ishido and Takaba [21] for the purpose of stability analysis of quantized feedback systems. Based on this framework, we will derive a sufficient condition for the small $\ell^{\infty}$ signal $\ell^{\infty}$ stability of the NCS in terms of the number of quantization levels of the quantizer and the maximum number of consecutive packet-dropouts.

The results of the present work complement those of $[15,17,18]$ by taking into account perturbations and quantization effects, and also those presented 
recently in [16]. In the latter work, the controller is based upon model predictive control ideas, and no quantization effects were considered.

The remainder of this manuscript is organized as follows: In Section 2, we describe the NCS to be studied. Section 3 revises the notion of small $\ell^{\infty}$ signal $\ell^{\infty}$ stability. In Section 4, we derive a linear fractional transformation model of the NCS. This model is used, in Section 5, to derive sufficient conditions for stability of the NCS. A numerical example is included in Section 6. Section 7 draws conclusions.

Notation. The sets of real numbers, positive integers, and non-negative integers are denoted via $\mathbb{R}, \mathbb{N}$, and $\mathbb{Z}_{+}$, respectively.

The $\infty$-norm of a vector $x \in \mathbb{R}^{n}$ is given by $\|x\|_{\infty}=\max _{1 \leq i \leq n}\left|x_{i}\right|$. We also define the 1-norm of a matrix $A \in \mathbb{R}^{n \times m}$ by $\|A\|_{1}:=\max _{1 \leq i \leq n} \sum_{j=1}^{m}\left|a_{i j}\right|$, where $a_{i j}$ is the $(i, j)$-th component of $A$.

The function space $\ell^{\infty}$ is defined by

$$
\ell^{\infty}:=\left\{f: \mathbb{Z}_{+} \rightarrow \mathbb{R}^{n} \mid \sup _{t \in \mathbb{Z}_{+}}\|f(t)\|_{\infty}<\infty\right\}
$$

This function space is equipped with the norm $\|f\|_{\ell^{\infty}}:=\sup _{t \in \mathbb{Z}_{+}}\|f(t)\|_{\infty}$.

If a map $H$ is unbiased finite gain $\ell^{\infty}$ stable, its $\ell^{\infty}$ gain is denoted by $\|H\|_{\ell \infty-\text {-ind }}$, i.e.,

$$
\|H\|_{\ell^{\infty} \text {-ind }}=\inf \left\{\gamma>0 \mid\|H u\|_{\ell^{\infty}} \leq \gamma\|u\|_{\ell^{\infty}} \forall u \in \ell^{\infty}\right\} .
$$

\section{System Description}

We consider a NCS with an unreliable communication channel affected by packet-dropouts, as depicted in Fig. 1. To alleviate the effect of packetdropouts, the NCS incorporates a buffering mechanism in the feedback loop (see, e.g., $[15,16]$ ). However, unlike the setting studied in $[15,16]$, we here explicitly take quantization effects into account. More precisely, the encodercontroller sends out quantized values of current and finite step future control signals at each time instant. We will give a detailed system description below. Plant $\boldsymbol{P}: \quad$ The plant $P$ is a discrete-time linear time-invariant system whose state-space representation is given by

$$
x(t+1)=A x(t)+B u(t)+w(t), \quad t \in \mathbb{N} .
$$




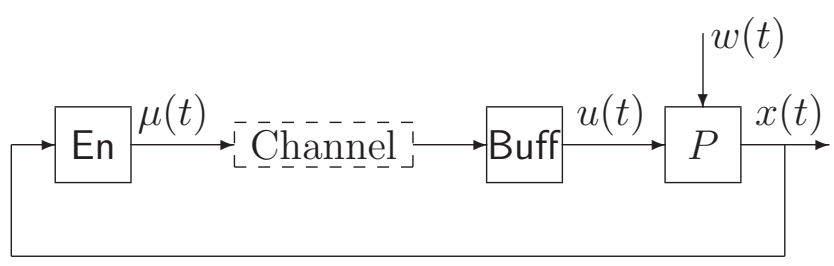

Figure 1: NCS with quantized control values and packet-dropouts

The signals $x(t) \in \mathbb{R}^{n}, u(t) \in \mathbb{R}$ and $w(t) \in \mathbb{R}^{n}$ are the plant state, the actuator input and the process disturbance, respectively. The initial state $x(0)$ is assumed to be zero.

Channel: The communication channel is affected by packet-dropouts. The packet-dropout is characterized in terms of the discrete variable $s(t)$ defined by

$$
s(t)=\left\{\begin{array}{l}
1 \text { if a packet-dropout does not occur at time } t \\
0 \text { if a packet-dropout occurs at time } t
\end{array}\right.
$$

Whenever packet-dropouts do not occur, the channel transmits the current control packet to the buffer Buff without errors or delays.

In the sequel, we will denote the time instants when the transmission is successfully completed (i.e. a packet is not dropped) with $\left\{t_{0}, t_{1}, t_{2}, \cdots\right\}$, and assume $t_{0}=0$. We thus have

$$
s(t)=1 \Leftrightarrow\left(t=t_{i} \text { for some } i \in \mathbb{Z}_{+}\right) .
$$

Controller-Encoder En: Throughout this work, we will assume that no acknowledgments of receipt are available. Thus, the controller-encoder En does not know whether previous data packets were successfully received or not.

To compensate for possible future packet dropouts, at every time $t \in \mathbb{N}$ the controller-encoder transmits a control packet, say $\mu(t) \in \mathbb{R}^{N}$ to the buffer. The value $N \in \mathbb{N}$ is given and represents the packet size and also the buffer length. The control packet is composed of quantized potential control inputs for the current time instant and $N-1$ future time instants:

$$
\begin{aligned}
\mu(t) & =\left[\begin{array}{c}
q(\hat{u}(t ; t)) \\
q(\hat{u}(t+1 ; t)) \\
\vdots \\
q(\hat{u}(t+N-1 ; t))
\end{array}\right], \\
\hat{u}(t+i ; t) & =K \hat{x}(t+i ; t), \quad i \in\{0, \cdots, N-1\},
\end{aligned}
$$




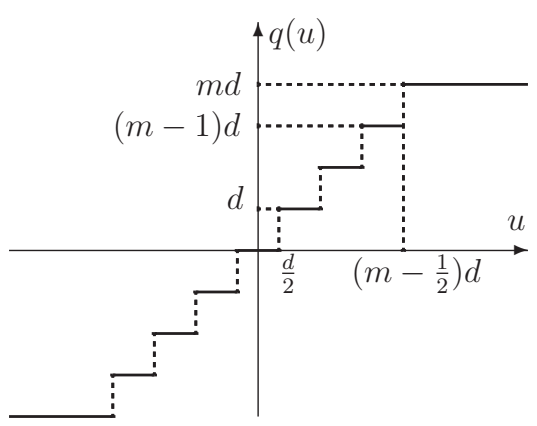

Figure 2: Uniform quantizer $q$

where $\hat{u}(t+i ; t) \in \mathbb{R}$ and $\hat{x}(t+i ; t) \in \mathbb{R}^{n}$ are the $i$-step predictions of the (unquantized) control input and the plant state based on the current state $x(t)$, respectively, and where $K \in \mathbb{R}^{1 \times n}$ is a static state-feedback gain.

In (4), the function $q: \mathbb{R} \rightarrow \mathbb{V}:=\{0, \pm d, \pm 2 d, \cdots, \pm m d\}$ denotes a uniform static quantizer, where $d \in \mathbb{R}$ and $m \in \mathbb{N}$ are positive constants. As shown in Fig. 2, the quantizer produces a quantized symbol by rounding its input to the nearest discrete value in $\mathbb{V}$ :

$$
q(u)=\left\{\begin{array}{cc}
m d, & \text { if }\left(m-\frac{1}{2}\right) d \leq u, \\
(m-1) d, & \text { if }\left(m-\frac{3}{2}\right) d \leq u<\left(m-\frac{1}{2}\right) d, \\
\vdots & \vdots \\
0, & \text { if }-\frac{1}{2} d \leq u<\frac{1}{2} d, \\
\vdots & \vdots \\
-m d, & \text { if } u<-\left(m-\frac{1}{2}\right) d .
\end{array}\right.
$$

The constant $d$ is the step size, whereas $M:=2 m+1$ is the number of quantization levels. ${ }^{1}$

The state predictions $\hat{x}(t+i ; t)(i=1,2, \cdots, N-1)$ are calculated recursively based on the current state $x(t)$ and the plant dynamics as follows:

$$
\hat{x}(t+i ; t)=\left\{\begin{array}{cl}
x(t), & \text { if } i=0, \\
A \hat{x}(t+i-1 ; t)+B q(\hat{u}(t+i-1 ; t)), & \text { if } i=1, \cdots, N-1 .
\end{array}\right.
$$

\footnotetext{
${ }^{1}$ Due to quantization, each control packet $\mu(t)$ can take only one of $M^{N}$ different values, and can, thus, be expressed via $N \log _{2} M$ bits.
} 
Buffer Buff: The buffer Buff provides the actuator values based on the received channel symbols. The state of the buffer is updated every time it successfully receives the packet $\mu$. To be more precise, the dynamics of Buff is described by

$$
b(t)=s(t) \mu(t)+(1-s(t)) S b(t-1), \quad b(-1)=0,
$$

where $b(t) \in \mathbb{R}^{N}$ denotes the state of Buff, and

$$
S:=\left[\begin{array}{ccccc}
0 & 1 & 0 & \cdots & 0 \\
\vdots & \ddots & \ddots & \ddots & \vdots \\
0 & \cdots & 0 & 1 & 0 \\
0 & \cdots & \cdots & 0 & 1 \\
0 & \cdots & \cdots & \cdots & 0
\end{array}\right] \in \mathbb{R}^{N \times N}
$$

is a shift matrix. Then, the plant input $u(t)$ is given by

$$
u(t)=\left[\begin{array}{llll}
1 & 0 & \cdots & 0
\end{array}\right] b(t)
$$

see Fig. 1.

The buffering technique adopted here was used, e.g., in [16] to study Input-to-State Stability [14] of a related NCS, where the control packets are designed without quantizations by adapting the model predictive control framework. In this paper, we complement [16] through the study of the NCS subject to packet-dropouts as well as finite-level quantization of control signals.

To put our subsequent analysis into context, it is worth recalling that, in the presence of finite-level quantization, finite gain $\ell^{p}$ stability of the closedloop system cannot be established [13]. Thus, in this paper, we are interested in a weaker version of stability, namely small $\ell^{\infty}$ signal $\ell^{\infty}$ stability. This notion was introduced in $[19,20]$ and is briefly revised in the following section.

\section{Small $\ell^{\infty}$ Signal $\ell^{\infty}$ Stability}

We briefly revise some basic results on small $\ell^{\infty}$ signal $\ell^{\infty}$ stability which was introduced in $[19,20]$.

Definition 1. (small $\ell^{\infty}$ signal $\ell^{\infty}$ stability) $A$ map $H$ is said to be small $\ell^{\infty}$ signal $\ell^{\infty}$ stable if there exist positive constants $\epsilon$ and $\gamma$ such that

$$
\llbracket\|u\|_{\ell^{\infty}} \leq \epsilon \Rightarrow\|H u\|_{\ell^{\infty}} \leq \gamma \epsilon \rrbracket, \quad \forall u \in \ell^{\infty} .
$$




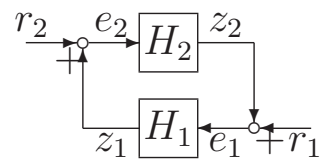

Figure 3: Feedback system for stability analysis

The quantities $\gamma$ and $\epsilon$ are called the attenuation level and the input bound, respectively.

The feedback system in Fig. 3 is called small $\ell^{\infty}$ signal $\ell^{\infty}$ stable if there exist positive constants $\epsilon$ and $\gamma$ such that

$$
\|\|\left[\begin{array}{l}
r_{1} \\
r_{2}
\end{array}\right]\left\|_{\ell^{\infty}} \leq \epsilon \Rightarrow\right\|\left[\begin{array}{l}
z_{1} \\
z_{2}
\end{array}\right] \|_{\ell^{\infty}} \leq \gamma \epsilon \rrbracket, \quad \forall r_{1}, r_{2} \in \ell^{\infty}
$$

We next briefly summarize some connections between the small $\ell^{\infty}$ signal $\ell^{\infty}$ notion and other stability notions:

Remark 1. (i) The small $\ell^{\infty}$ signal $\ell^{\infty}$ stability in Definition 1 is the local version of the $\ell^{\infty}$ stability (see Definition 2 in Appendix A). In fact, the small $\ell^{\infty}$ signal $\ell^{\infty}$ stability implies $\ell^{\infty}$ boundedness of the output only for the inputs with small $\ell^{\infty}$ norm while $\ell^{\infty}$ stability guarantees the $\ell^{\infty}$ boundedness of the output for all $\ell^{\infty}$ inputs.

(ii) Although the small $\ell^{\infty}$ signal $\ell^{\infty}$ stability is, in general, a weaker notion than the traditional $\ell^{\infty}$ stability as described above, in the special case of linear maps, these stability notions are equivalent (see Theorem 1 in [21]).

(iii) The small $\ell^{\infty}$ signal $\ell^{\infty}$ stability notion differs from finite gain $\ell^{\infty}$ stability[22] and local $\ell^{\infty}$ stability [23] (see Definition 3 and 4 in Appendix A for the definitions of these notions), in that it does not use the notion of $\ell^{\infty}$ gains. Instead, the attenuation level of a map, is expressed in terms of the ratio of the local upper bounds of the $\ell^{p}$ norms of input-output signals.

In $[19,20]$, the current authors derived a sufficient condition for small $\ell^{\infty}$ signal $\ell^{\infty}$ stability of the feedback system in Fig. 3. For our current purposes, these conditions can be summarized as follows: 


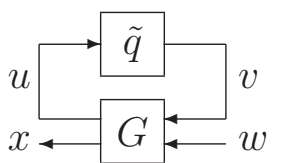

Figure 4: Linear fractional representation of the NCS

Proposition 1 ([19, 20]). Consider the feedback system in Fig. 3, and assume that the following three conditions hold:

(i) $H_{1}: e_{1} \mapsto z_{1}$ is strictly causal and is unbiased finite $\ell^{\infty}$ stable with gain not greater than a positive constant $\gamma_{1}$, namely

$$
\|z\|_{\ell^{\infty}} \leq \gamma_{1}\left\|e_{1}\right\|_{\ell^{\infty}}, \quad \forall e_{1} \in \ell^{\infty}
$$

(ii) For $\mathrm{H}_{2}: e_{2} \mapsto z_{2}$, there exist positive constants $\epsilon_{2}$ and $\gamma_{2}$ such that

$$
\llbracket\left\|e_{2}\right\|_{\ell^{\infty}} \leq \epsilon_{2} \Rightarrow\left\|z_{2}\right\|_{\ell^{\infty}} \leq \gamma_{2} \epsilon_{2} \rrbracket, \quad \forall e_{2} \in \ell^{\infty} .
$$

(iii) $\gamma_{1} \gamma_{2}<1$.

Then, the feedback system is small $\ell^{\infty}$ signal $\ell^{\infty}$ stable. In particular,

$$
\llbracket\left\|\left[\begin{array}{l}
r_{1} \\
r_{2}
\end{array}\right]\right\|_{\ell^{\infty}} \leq \epsilon \Rightarrow\left(\left\|z_{1}\right\|_{\ell^{\infty}} \leq \delta_{1} \text { and }\left\|z_{2}\right\|_{\ell^{\infty}} \leq \delta_{2}\right) \rrbracket, \quad \forall r \in \ell^{\infty}
$$

holds for

$$
\epsilon=\frac{\left(1-\gamma_{1} \gamma_{2}\right) \epsilon_{2}}{1+\gamma_{1}}, \quad \delta_{1}=\frac{\left(1+\gamma_{2}\right) \gamma_{1} \epsilon_{2}}{1+\gamma_{1}}, \quad \delta_{2}=\gamma_{2} \epsilon_{2}
$$

\section{Linear fractional transformation model of the NCS}

The NCS in Fig. 1 is nonlinear and time-varying due to quantization, packet-dropouts, and buffering. To study the stability of the NCS, we will first extract the nonlinearity (denoted by $\tilde{q}$ ) associated with the quantization error to re-formulate the feedback system of Fig. 1 as a linear fractional transformation of Fig. 4, where the "nominal" subsystem $G$ is linear and time-varying. 
It follows from (3), (4), (8), (9) and (10) that the plant inputs at the time instants

$$
\mathbb{N}=\cup_{i \in \mathbb{N}}\left\{t_{i}, t_{i}+1, \ldots, t_{i+1}-1\right\}
$$

are given by

$$
u(t)=q\left(\hat{u}\left(t ; t_{i}\right)\right), \quad t \in\left\{t_{i}, t_{i}+1, \ldots, t_{i+1}-1\right\} .
$$

Thus, the plant model (1) can be rewritten as

$$
x(t+1)=A x(t)+B q\left(\hat{u}\left(t ; t_{i}\right)\right)+w(t), t \in\left\{t_{i}, t_{i}+1, \ldots, t_{i+1}-1\right\} .
$$

If we denote the quantization error at each time instant $t \in \mathbb{N}$ via

$$
v(t):=q\left(\hat{u}\left(t ; t_{i}\right)\right)-\hat{u}\left(t ; t_{i}\right), \quad t \in\left\{t_{i}, t_{i}+1, \ldots, t_{i+1}-1\right\},
$$

then it follows from (7) and (17) that

$$
\begin{aligned}
\hat{x}\left(t+1 ; t_{i}\right) & =A \hat{x}\left(t ; t_{i}\right)+B\left(\hat{u}\left(t ; t_{i}\right)+v(t)\right) \\
& =A_{K} \hat{x}\left(t ; t_{i}\right)+B v(t), \quad t \in\left\{t_{i}, t_{i}+1, \ldots, t_{i+1}-2\right\},
\end{aligned}
$$

Moreover, (7) and (16) give that

$$
x(t)=\hat{x}\left(t ; t_{i}\right)+\sum_{l=1}^{t-t_{i}} A^{l-1} w(t-l), \quad t \in\left\{t_{i}, t_{i}+1, \ldots, t_{i+1}-1\right\},
$$

and thus,

$$
\begin{aligned}
\hat{x}\left(t_{i+1} ; t_{i+1}\right) & =x\left(t_{i+1}\right) \\
& =\hat{x}\left(t_{i+1} ; t_{i}\right)+\sum_{l=1}^{t_{i+1}-t_{i}} A^{l-1} w\left(t_{i+1}-l\right) \\
& =A_{K} \hat{x}\left(t_{i+1}-1 ; t_{i}\right)+B v\left(t_{i+1}-1 ; t_{i}\right) \\
& \quad+\sum_{l=1}^{t_{i+1}-t_{i}} A^{l-1} w\left(t_{i+1}-l\right), \quad i \in \mathbb{N} .
\end{aligned}
$$

It follows from (5), (18), (19) and (20) that the NCS in Fig. 1 can be described in terms of the linear fractional transformation of a linear subsystem $G$ and the static nonlinear function $\tilde{q}$, as depicted in Fig. 4. 
The nonlinearity $\tilde{q}$ is a static map defined by

$$
\tilde{q}(\hat{u})=q(\hat{u})-\hat{u},
$$

and, thus, represents the quantization error, as illustrated in Fig. 5. It can be easily verified from (6) that $\tilde{q}$ satisfies

$$
\llbracket\|\hat{u}\|_{\ell^{\infty}} \leq \frac{M d}{2} \Rightarrow\|\tilde{q}(\hat{u})\|_{\ell^{\infty}} \leq \frac{d}{2} \rrbracket, \quad \forall \hat{u} \in \ell^{\infty} .
$$

In other words, $\tilde{q}$ has small $\ell^{\infty}$ signal $\ell^{\infty}$ stability with attenuation level $1 / M$ and input bound $M d / 2$ (see Definition 1 in Section 3).

To characterize the subsystem $G$ in Fig. 4 , it is convenient to introduce a state vector $\xi(t)$ and control signal $\hat{u}(t)$ via:

$$
\xi(t):=\hat{x}\left(t ; t_{i}\right), \quad \hat{u}(t):=\hat{u}\left(t ; t_{i}\right), \quad t \in\left\{t_{i}, t_{i}+1, \ldots, t_{i+1}-1\right\}, i \in \mathbb{N}
$$

This allows us to describe $G$ in state-space form via

$$
G:\left\{\begin{aligned}
\xi(t+1) & =A_{K} \xi(t)+B v(t)+F_{1} w(t), \\
\hat{u}(t) & =K \xi(t), \\
x(t) & =\xi(t)+F_{2} w(t),
\end{aligned}\right.
$$

where $F_{1}$ and $F_{2}$ are linear time-varying maps defined by

$$
\begin{aligned}
& F_{1}: w \mapsto\left\{\begin{array}{cl}
0, & \text { if } t \in\left\{t_{i}, t_{i}+1, \ldots, t_{i+1}-2\right\}, \\
\sum_{l=1}^{t_{i+1}-t_{i}} A^{l-1} w\left(t_{i+1}-l\right), & \text { if } t=t_{i+1}-1,
\end{array}\right. \\
& F_{2}: w \mapsto\left\{\begin{array}{cl}
0, & \text { if } t=t_{i}, \\
\sum_{l=1}^{t-t_{i}} A^{l-1} w(t-l), & \text { if } t \in\left\{t_{i}+1, t_{i}+2, \ldots, t_{i+1}-1\right\} .
\end{array}\right.
\end{aligned}
$$

Interestingly, the map $G$ : $(v, w) \mapsto(u, x)$ can be decomposed as

$$
G=\left[\begin{array}{cc}
G_{00} & G_{01} \circ F_{1} \\
G_{10} & G_{11} \circ F_{1}+F_{2}
\end{array}\right],
$$

where $G_{00}, G_{01}, G_{10}$ and $G_{11}$ are LTI maps with impulse responses

$$
\begin{aligned}
& g_{00}(t)=\left\{\begin{array}{cl}
0, & \text { if } t=0, \\
K A_{K}^{t-1} B, & \text { if } t \in \mathbb{N},
\end{array} \quad g_{01}(t)=\left\{\begin{array}{cc}
0, & \text { if } t=0, \\
K A_{K}^{t-1}, & \text { if } t \in \mathbb{N},
\end{array},\right.\right. \\
& g_{10}(t)=\left\{\begin{array}{cl}
0, & \text { if } t=0, \\
A_{K}^{t-1} B, & \text { if } t \in \mathbb{N},
\end{array} \quad g_{11}(t)=\left\{\begin{array}{cc}
0, & \text { if } t=0, \\
A_{K}^{t-1}, & \text { if } t \in \mathbb{N},
\end{array}\right.\right.
\end{aligned}
$$




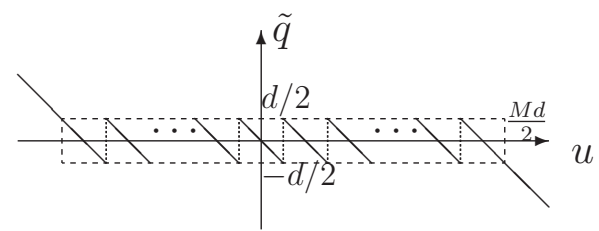

Figure 5: Nonlinearity associated with quantization error

respectively. It is easily seen from these equations that the effect of packetdropouts is confined in the time-varying maps $F_{1}$ and $F_{2}$.

\section{Stability Analysis of the Networked Control System}

With Section 4 as a background, analyzing stability of the NCS gives rise to the problem of studying small $\ell^{\infty}$ signal $\ell^{\infty}$ stability of the closed-loop map from $w$ to $x$ in Fig. 1. For this purpose, we will make the following assumptions:

Assumption 1. The number of consecutive packet-dropouts is bounded by the buffer length $N$, i.e., we have:

$$
1 \leq t_{i+1}-t_{i} \leq N, \quad \forall i \in \mathbb{N}
$$

Assumption 2. The matrix $A_{K}:=A+B K$ is Schur stable.

If Assumption 1 is satisfied, then the buffer length $N$ amounts to the maximal number of consecutive packet-dropouts. Assumption 2 implies that the controller gain $K$ in (5) stabilizes the plant model (1) in the absence of dropouts or quantization constraints.

Now, we are in a position to state our stability analysis problem in precise terms: ${ }^{2}$

\footnotetext{
${ }^{2}$ Note that the condition (29) is different from the small $\ell^{\infty}$ signal $\ell^{\infty}$ stability of the feedback system in Fig. 3.
} 
Under Assumptions 1 and 2, we wish to derive a sufficient condition for the existence of positive constants $\epsilon$ and $\gamma$ such that

$$
\llbracket\|w\|_{\ell^{\infty}} \leq \epsilon \Rightarrow\|x\|_{\ell^{\infty}} \leq \gamma \epsilon \rrbracket, \quad \forall w \in \ell^{\infty}
$$

for any sequences of packet-dropouts satisfying Assumption 1. Furthermore, if the stability condition is satisfied, we wish to characterize the relation (trade-off) between the attenuation level $\gamma$ and the parameters $M, d$ and $N$.

We will hereafter use the linear fractional representation derived in the previous section to obtain a sufficient condition for achieving (29) for the NCS in Fig. 1 (equivalently in Fig. 4).

\subsection{Preliminaries}

A key property of the linear fractional transformation model presented in Section 4 is that, if Assumption 2 holds, then the LTI maps $G_{00}, G_{01}$, $G_{10}$ and $G_{11}$ are stable and have finite $\ell^{\infty}$ gains $\left(\ell^{1}\right.$ norms of their impulse responses). Furthermore, it is easy to see that $F_{1}$ and $F_{2}$ also have finite $\ell^{\infty}$ gains:

Lemma 1. Suppose that Assumption 1 holds. Then, the maps $F_{1}$ and $F_{2}$ defined in (25) and (26) are unbiased finite gain $\ell^{\infty}$ stable, and

$$
\left\|F_{1}\right\|_{\ell^{\infty} \text {-ind }} \leq \kappa, \quad\left\|F_{2}\right\|_{\ell^{\infty} \text {-ind }} \leq \kappa
$$

hold for

$$
\kappa:=\sum_{l=0}^{N-1}\left\|A^{l}\right\|_{1}<\infty .
$$

Proof. See Appendix B.

As we can see, the linear fractional transformation model provides important information for the stability analysis of the NCS in Fig. 1.

Firstly, by virtue of the buffering technique, the adverse effect of packetdropouts is isolated from the feedback loop. Indeed, the maximal length $N$ of consecutive packet-dropouts is contained only in the stable feedforward maps $F_{1}$ and $F_{2}$. This implies that packet-dropouts play no role in deteriorating stability of the NCS. 


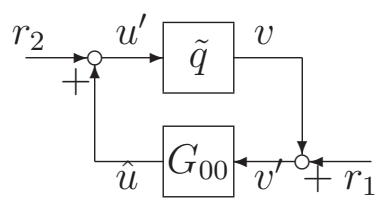

Figure 6: Feedback interconnection of $G_{00}$ and $\tilde{q}$

Secondly, since $G_{00}$ is a strictly causal map and $\tilde{q}$ is a static nonlinear function, the feedback system in Fig. 4 is well-posed. Thus, we can define the closed-loop map from $w$ to $x$.

\subsection{Main Results}

Lemma 2 stated below shows that, since all 4 components of $G$ are finite gain $\ell^{\infty}$ stable, the stability analysis of the overall NCS reduces to investigating the stability of the feedback interconnection between $G_{00}$ and $\tilde{q}$ in Fig. 6 . To state our result, we denote the $\ell^{\infty}$ gains of the components of $G$ via

$$
\begin{aligned}
\gamma_{00}:=\left\|G_{00}\right\|_{\ell^{\infty} \text {-ind }}, & \gamma_{01}:=\left\|G_{01}\right\|_{\ell^{\infty} \text {-ind }}, \\
\gamma_{10}:=\left\|G_{10}\right\|_{\ell^{\infty} \text {-ind }}, & \gamma_{11}:=\left\|G_{11}\right\|_{\ell^{\infty} \text {-ind }} .
\end{aligned}
$$

Lemma 2. Assume that the feedback interconnection of $G_{00}$ and $\tilde{q}$ in Fig. 6 is small $\ell^{\infty}$ signal $\ell^{\infty}$ stable, i.e., there exist positive constants $\epsilon_{r}, \delta_{u} \delta_{v}$ such that

$$
\left.\llbracket\left\|\left[\begin{array}{l}
r_{1} \\
r_{2}
\end{array}\right]\right\|_{\ell^{\infty}} \leq \epsilon_{r} \Rightarrow\left(\|\hat{u}\|_{\ell^{\infty}} \leq \delta_{u} \text { and }\|v\|_{\ell^{\infty}} \leq \delta_{v}\right)\right], \quad \forall r_{1}, r_{2} \in \ell^{\infty} .
$$

Then, the feedback system in Fig. 4 satisfies

$$
\llbracket\|w\|_{\ell^{\infty}} \leq \epsilon \Rightarrow\|x\|_{\ell^{\infty}} \leq \gamma \epsilon \rrbracket, \quad \forall w \in \ell^{\infty}
$$

for any sequences of packet-dropouts satisfying Assumption 1, where

$$
\epsilon=\frac{\epsilon_{r}}{\gamma_{01} \kappa}, \quad \gamma=\frac{\gamma_{01} \kappa\left(\left(\gamma_{11}+1\right) \epsilon_{r} \kappa+\gamma_{10} \delta_{v}\right)}{\epsilon_{r}},
$$

and $\kappa$ is defined in (31). 
Proof. From (27), the input-output relations in Fig. 4 are described by

$$
\begin{aligned}
& v=\tilde{q}(\hat{u}), \\
& \hat{u}=G_{00} v+\left(G_{01} \circ F_{1}\right) w, \\
& x=G_{10} v+\left(G_{11} \circ F_{1}+F_{2}\right) w .
\end{aligned}
$$

On the other hand, Lemma 1 gives

$$
\begin{aligned}
& \left\|G_{01} \circ F_{1}\right\|_{\ell^{\infty} \text {-ind }} \leq\left\|G_{01}\right\|_{\ell^{\infty} \text {-ind }}\left\|F_{1}\right\|_{\ell^{\infty} \text {-ind }} \leq \gamma_{01} \kappa \\
& \left\|G_{11} \circ F_{1}+F_{2}\right\|_{\ell^{\infty} \text {-ind }} \leq\left\|G_{11}\right\|_{\ell^{\infty} \text {-ind }}\left\|F_{1}\right\|_{\ell^{\infty} \text {-ind }}+\left\|F_{2}\right\|_{\ell^{\infty} \text {-ind }} \leq \gamma_{11} \kappa+\kappa .
\end{aligned}
$$

It is easily seen by taking $r_{1}:=0, r_{2}:=\left(G_{01} \circ F_{1}\right) w, u^{\prime}:=u$ in Fig. 6 that the small $\ell^{\infty}$ signal $\ell^{\infty}$ stability of the feedback interconnection $\left(G_{00}, \tilde{q}\right)$ implies that

$$
\left\|G_{01} \circ F_{1} w\right\|_{\ell^{\infty}} \leq \epsilon_{r} \Rightarrow\left(\|\hat{u}\|_{\ell^{\infty}} \leq \delta_{u} \text { and }\|v\|_{\ell^{\infty}} \leq \delta_{v}\right)
$$

holds for the feedback system of (36)-(38) in Fig. 4.

Now, we assume that $\|w\|_{\ell^{\infty}} \leq \epsilon$ where $\epsilon$ is defined by (35). To complete the proof, we only need to establish boundedness of $\|x\|_{\ell_{\infty}}$.

It follows from (39) that

$$
\left\|G_{01} \circ F_{1} w\right\|_{\ell^{\infty}} \leq\left\|G_{01} \circ F_{1}\right\|_{\ell^{\infty} \text {-ind }}\|w\|_{\ell^{\infty}} \leq \gamma_{01} \kappa \epsilon=\epsilon_{r} .
$$

We then have $\|v\|_{\ell^{\infty}} \leq \delta_{v}$ from (41) and (42). Therefore, we conclude from (38) and (40) that

$$
\begin{aligned}
\|x\|_{\ell^{\infty}} & \leq\left\|G_{00}\right\|_{\ell^{\infty} \text {-ind }}\|v\|_{\ell^{\infty}}+\left\|G_{11} \circ F_{1}+F_{2}\right\|_{\ell^{\infty} \text {-ind }}\|w\|_{\ell^{\infty}} \\
& \leq \gamma_{00} \delta_{v}+\left(\gamma_{11}+1\right) \kappa \epsilon \\
& =\gamma \epsilon<+\infty
\end{aligned}
$$

where $\gamma:=\gamma_{00} \delta_{v} / \epsilon+\left(\gamma_{11}+1\right) \kappa$.

Having established Lemma 2, we will next present the main result of this paper, namely a sufficient condition for the small $\ell^{\infty}$ signal $\ell^{\infty}$ stability of the closed-loop map from $w$ to $x$ in Fig. 4. Our result, stated in Theorem 1, also quantifies the disturbance attenuation level in terms of the parameters of the quantizer and the buffer length. 
Theorem 1. Suppose that Assumptions 1 and 2 hold. If

$$
\gamma_{00}<M
$$

then the NCS in Fig. 1 satisfies

$$
\llbracket\|w\|_{\ell^{\infty}} \leq \epsilon \Rightarrow\|x\|_{\ell^{\infty}} \leq \gamma \epsilon \rrbracket \quad \forall w \in \ell^{\infty}
$$

for any packet-dropout sequences satisfying Assumption 1, where

$$
\begin{aligned}
\epsilon & =\frac{\left(M-\gamma_{00}\right) d}{2 \gamma_{01}\left(\gamma_{00}+1\right) \kappa}, \\
\gamma & =\frac{\gamma_{01} \kappa\left(\gamma_{10}\left(\gamma_{00}+1\right)+\left(\gamma_{11}+1\right)\left(M-\gamma_{00}\right) \kappa\right)}{M-\gamma_{00}} .
\end{aligned}
$$

Proof. Suppose that (44) holds. Since $G_{00}$ is strictly causal, it follows from (44), (22), and Proposition 1 that the feedback interconnection of $G_{00}$ and $\tilde{q}$ in Fig. 6 is small $\ell^{\infty}$ signal $\ell^{\infty}$ stable. In particular, we have

$$
\llbracket\|r\|_{\ell^{\infty}} \leq \epsilon_{r} \Rightarrow\left(\|u\|_{\ell^{\infty}} \leq \delta_{u} \text { and }\|v\|_{\ell^{\infty}} \leq \delta_{v}\right) \rrbracket, \quad \forall r \in \ell^{\infty},
$$

where the constants

$$
\epsilon_{r}=\frac{\left(M-\gamma_{00}\right) d}{2\left(1+\gamma_{00}\right)}, \quad \delta_{u}=\frac{(M+1) \gamma_{00} d}{2\left(1+\gamma_{00}\right)}, \quad \delta_{v}=\frac{d}{2}
$$

are obtained by substituting $\gamma_{1}:=\gamma_{00}, \gamma_{2}:=1 / M$ and $\epsilon_{2}:=M d / 2$ into (14).

Consequently, we conclude from Lemma 2 that the NCS in Fig. 1, equivalently Fig. 4, satisfies (45), where $\epsilon$ and $\gamma$ in (46), (47) are obtained by substituting (48) into (35).

Theorem 1 establishes that small $\ell^{\infty}$ signal $\ell^{\infty}$ stability of the closed-loop map from $w$ to $x$ in the presence of bounded packet dropouts and finite level quantization can be guaranteed if a large enough number of quantization levels $M$ is available. Whilst the condition (44) does not depend on the step size $d$, this quantizer parameter does affect the input bound $\epsilon$ in (46). Since the input bound $\epsilon$ is monotonically increasing with respect to $d$, compensating for a large disturbance $w(t)$ requires a large step size $d$. This observation is rather intuitive, since, with large $d$, the control signal is allowed to take large values as shown in (6). Note also that the upper bound on $\|x\|_{\ell^{\infty}}$ also depends on $d$ through the input bound $\epsilon$ in (34). 
Our stability condition (44) also indicates that stability of the closedloop map from $w$ to $x$ is independent of the maximum number of consecutive packet dropouts $N$, provided sufficient control inputs are contained in each control packet, see Assumption 1. However, the disturbance attenuation level of the closed-loop map from $w$ to $x$ is strongly affected by $N$. In fact, it can be seen from (31) and (47) that, for open-loop unstable plants, the attenuation level $\gamma$ is exponentially increasing with respect to $N$. This suggests that the magnitude of the state $x$ may become extremely large, if the network introduces too many consecutive packet-dropouts. The latter observation is hardly surprising, since, during periods of consecutive dropouts, the plant is unavoidably left in open loop.

\section{Numerical Examples}

\subsection{Example 1 (scalar plant)}

Consider a scalar plant described by

$$
x(t+1)=a x(t)+u(t)+w(t), \quad a>1 .
$$

We choose the stabilizing (deadbeat) feedback gain $K=-a$, providing $A_{K}=$ $A+B K=0$. In this case, the $\ell^{\infty}$ gains in (32) are given by

$$
\gamma_{00}=a, \quad \gamma_{01}=a, \quad \gamma_{10}=1, \quad \gamma_{11}=1
$$

and the sufficient stability condition (44) becomes

$$
M>a .
$$

It then follows from (31), (46) and (47) that the parameters $\kappa, \epsilon$ and $\gamma$ are given by

$$
\begin{aligned}
\kappa & =\sum_{l=0}^{N-1} a^{l}=\frac{a^{N}-1}{a-1} \\
\epsilon & =\frac{(M-a) d}{2(a+1) \kappa}=\frac{(M-a) d}{2(a+1)} \frac{a-1}{a^{N}-1}, \\
\gamma & =\frac{a \kappa((a+1)+2(M-a) \kappa)}{M-a}=\frac{a(a+1)}{M-a} \frac{a^{N}-1}{a-1}+2 a\left(\frac{a^{N}-1}{a-1}\right)^{2} .
\end{aligned}
$$




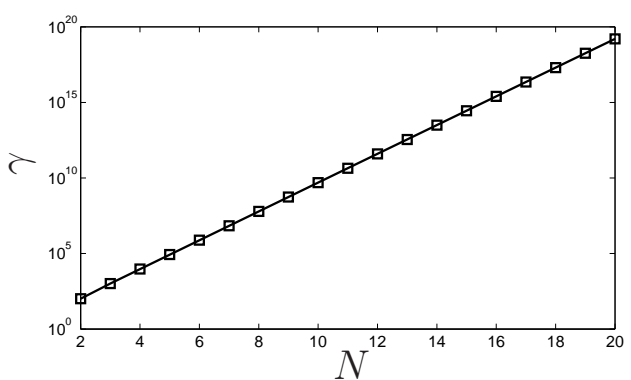

Figure 7: Effect of the maximal number of consecutive dropouts $N$ on the attenuation level $\gamma$

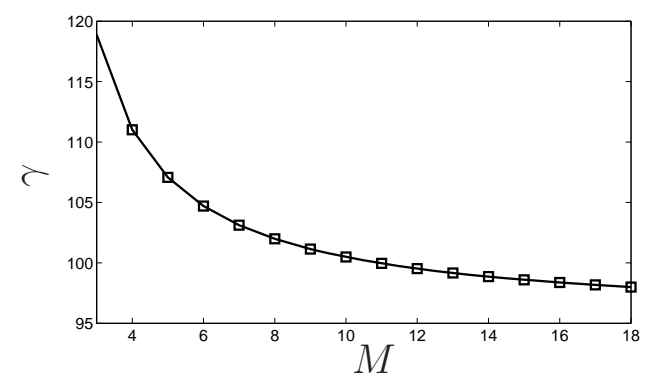

Figure 8: Trade-off between the attenuation level $\gamma$ and the number of quantization levels $M$

Interestingly, it turns out that, for scalar plant models, the stability condition (49) is tight. In fact, it is known that $M \geq a$ is a necessary condition to stabilize the scalar system over a rate-limited channel, even when there are no packet-dropouts $(N=1)$; see, e.g., [3].

As a special case, suppose that $a=2.99$. Then, the stability condition (49) becomes $M>2.99$, which corresponds to a quantizer with at least three levels. For this fixed $a$, the associated trade-off between the attenuation level $\gamma$ in (52) and the maximal number of consecutive packet-dropouts $N$ for a quantizer with $M=11$ quantization levels is illustrated in Fig. 7. As can be seen from that figure and (52), for a fixed $M, \gamma$ exponentially increases in $N$.

In Fig. 8, the relation between $\gamma$ and $M$ for fixed $N=2$ is illustrated. It can be seen from that figure that when $M$ is close to $\gamma_{00}=a=2.99$, the attenuation level $\gamma$ becomes very large. On the other hand, the attenuation level is monotonically decreasing with respect to $M$, and we have $\lim _{M \rightarrow \infty} \gamma=$ $2 a\left(a^{2}-1\right)^{2} /(a-1)^{2}=95.20$ as a lower bound of $\gamma$ in $(52)$. 


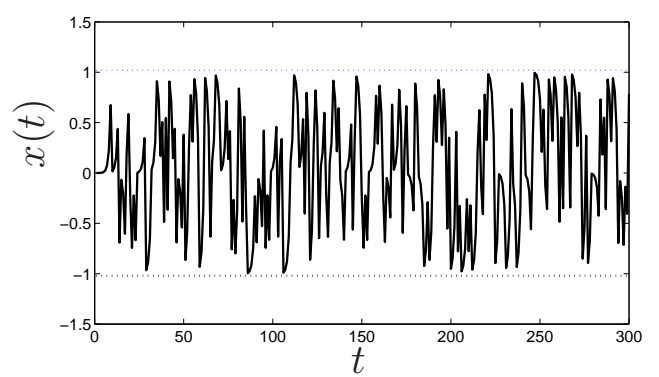

Figure 9: Plant state trajectory in the presence of dropouts and disturbances

We next performed a numerical simulation for a critical case where ${ }^{3}$

$$
\begin{aligned}
& w(t)=\epsilon, \forall t \in\{0,1, \ldots 300\} \\
& t_{i+1}-t_{i}=N=2, \quad \forall i \in\{0,1, \ldots 150\} .
\end{aligned}
$$

In this simulation, we choose $M=3, d=2$ and $N=2$. Then, it follows from (50) that $\kappa=3.99$. Theorem 1 then guarantees that the closed-loop map from $w$ to $x$ is small $\ell^{\infty}$ signal $\ell^{\infty}$ stable, and that (45) holds with

$$
\epsilon=2.1008 \times 10^{-4}, \quad \gamma=4.8553 \times 10^{3} .
$$

The simulation result of $x(t)$ for $t \in\{0,1, \ldots 300\}$ is depicted in Fig. 9, confirming (45), namely that $\|x(t)\|_{\infty}$ is less than $\delta:=\gamma \epsilon=1.02$ for all $t \in\{0,1, \ldots 300\}$.

\subsection{Example 2 (3rd-order plant)}

To verify the effectiveness of the buffering scheme considered in this paper, we next carry out simulations for the following three control schemes:

(a) Buffering scheme:

The buffering scheme considered in the previous sections is applied to compensate for packet-dropouts.

(b) Zero input scheme $(s(t)=0 \Rightarrow u(t)=0)$ :

If a packet is dropped, the actuator applies zero input to the plant.

\footnotetext{
${ }^{3}$ Namely, the disturbance takes the extreme value at each time instant, and the network periodically drops packets every other time instant.
} 


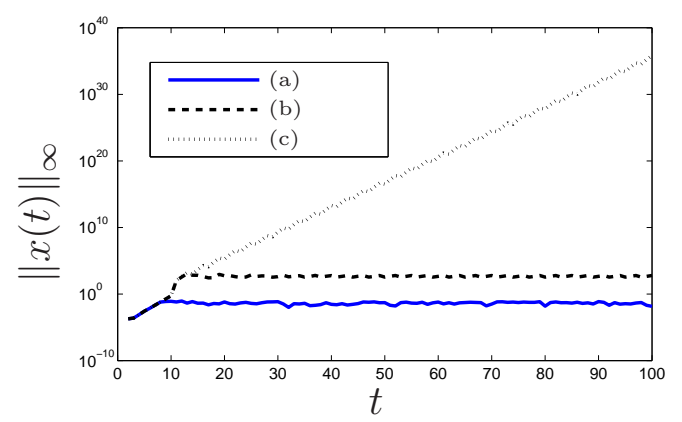

Figure 10: Simulation results for the control schemes (a), (b) and (c)

(c) Previous input scheme $(s(t)=0 \Rightarrow u(t)=u(t-1))$ :

If a packet is dropped, the actuator applies the previous input to the plant.

Consider the 3rd-order plant given by

$$
x(t+1)=\left[\begin{array}{ccc}
0.1 & 2.3 & 1.4 \\
0 & 2 & 1.5 \\
0 & 0.9 & 1.6
\end{array}\right] x(t)+\left[\begin{array}{c}
1.1 \\
0.9 \\
1
\end{array}\right] u(t)+w(t)
$$

and the communication channel with $N=3$. We choose the nominally stabilizing feedback gain as

$$
K=\left[\begin{array}{lll}
0.0013 & -1.6591 & -1.5782
\end{array}\right] .
$$

For this networked control system, we have $\kappa=42.63$ and

$$
\gamma_{00}=3.7545, \quad \gamma_{01}=4.3528, \quad \gamma_{10}=6.4171, \quad \gamma_{11}=7.4988 .
$$

We choose $M=5$ so that the stability condition (44) is satisfied. We carried out the simulations with random disturbances satisfying $\|w(t)\|_{\infty} \leq \epsilon=$ $5.8918 \times 10^{-4}$ and periodic packet-dropout sequences consisting of two consecutive dropouts and one success.

The simulation results are illustrated in Fig. 10. It is clearly seen from the figure that the buffering scheme (a) succeeds in keeping $\|x(t)\|_{\infty}$ much smaller compared with the zero input scheme (b) and (c). Furthermore, Fig. 10 shows that the previous input scheme (c) does not stabilize this plant with this particular choice of $K$ and $M$ because the plant state is unbounded. 
Note also that in the proposed scheme, it is sufficient to choose the feedback gain $K$, such that the matrix $A+B K$ is stable. This stands in stark contrast to the zero input scheme, where in the presence of packet-dropouts, a necessary condition for the stability of the NCS is that all finite products

$$
(A+B K) A^{i}, \quad i \in\{1, \cdots, N-1\}
$$

are stable. The class of admissible feedback gains is therefore quite restricted in the zero input case. On the other hand, in the proposed scheme the constraint on the feedback gain is much milder. This is useful when designing the networked control system.

\section{Concluding Remarks}

This paper has studied the small $\ell^{\infty}$ signal $\ell^{\infty}$ stability of a networked control system subject to disturbances, packet-dropouts and finite level quantization. By incorporating a buffering mechanism at the receiving end of the channel, the adverse effect of packet-dropouts on closed loop stability can be canceled as long as the number of consecutive dropouts is smaller than the buffer length. We have derived a sufficient condition for stability, which is stated in terms of the number of quantization levels. We have also elucidated the effect of the quantizer step size and the maximal number of consecutive packet-dropouts on the disturbance attenuation level. Numerical examples indicate that the scheme investigated can give significant performance gains when compared to alternative configurations.

Future work may include extending the approach to NCSs involving communication channels at both the actuator side and the sensor side, and also to NCSs which are subject to quantization, dropouts and delays.

\section{Acknowledgments}

The authors would like to thank Professors Yoshito Ohta and Hisaya Fujioka at Kyoto University for their helpful comments about this work. Dr. Quevedo is the recipient of an Australian Research Council Australian Research Fellowship (project number DP0988601).

\section{Appendix A. Basic stability notions}

This appendix collects the definitions of several stability notions referred to in this paper; see, e.g., [22]. 
Definition 2. ( $\ell^{\infty}$ stability)

$A$ map $H$ is $\ell^{\infty}$ stable if there exist a class $\mathcal{K}$ function $\alpha$ and a nonnegative constant $\beta$ such that

$$
\|H u\|_{\ell^{\infty}} \leq \alpha\left(\|u\|_{\ell^{\infty}}\right)+\beta, \quad \forall u \in \ell^{\infty} .
$$

In addition, $H$ is called unbiased if we can take $\beta=0$.

Definition 3. (finite gain $\ell^{\infty}$ stability)

A map $H$ is finite gain $\ell^{\infty}$ stable if there exist nonnegative constants $\gamma$ and $\beta$ such that

$$
\|H u\|_{\ell^{\infty}} \leq \gamma\|u\|_{\ell^{\infty}}+\beta, \quad \forall u \in \ell^{\infty} .
$$

The infimum of $\gamma$ satisfying the above inequality is called the $\ell^{\infty}$ gain of $H$.

Definition 4. (local $\ell^{\infty}$ stability) [23]

A map $H$ is locally $\ell^{\infty}$ stable if there exist nonnegative constants $\epsilon$ and $\gamma$ such that

$$
\llbracket\|u\|_{\ell^{\infty}} \leq \epsilon \Rightarrow\|H u\|_{\ell^{\infty}} \leq \gamma\|u\|_{\ell^{\infty}} \rrbracket, \quad \forall u \in \ell^{\infty} .
$$

\section{Appendix B. Proof of Lemma 1}

We give only the proof of $\left\|F_{1}\right\|_{\ell^{\infty} \text {-ind }} \leq \kappa$, since $\left\|F_{2}\right\|_{\ell^{\infty} \text {-ind }} \leq \kappa$ can be proved in the same manner.

Define $f_{1}=F_{1} w$ for $w \in \ell^{\infty}$. There always exists $i \in \mathbb{Z}_{+}$such that $t \in\left\{t_{i}, t_{i}+1, \cdots, t_{i+1}-1\right\}$, and $F_{1} w$ is given by $(25)$. Clearly, $\left\|f_{1}(t)\right\|_{\infty}=$ $0 \leq \kappa\|w\|_{\ell^{\infty}}$ holds for $t \in\left\{t_{i}, \cdots, t_{i+1}-2\right\}$. In the case of $t=t_{i+1}-1$, we obtain

$$
\begin{aligned}
\left\|f_{1}(t)\right\|_{\infty} & \leq \sum_{l=1}^{t_{i+1}-t_{i}}\left\|A^{l-1} w\left(t_{i+1}-l\right)\right\|_{\infty} \leq \sum_{l=1}^{t_{i+1}-t_{i}}\left\|A^{l-1}\right\|_{1}\left\|w\left(t_{i+1}-l\right)\right\|_{\infty} \\
& \leq \sum_{l=1}^{t_{i+1}-t_{i}}\left\|A^{l-1}\right\|_{1}\|w\|_{\ell^{\infty}} \leq \sum_{l=1}^{N}\left\|A^{l-1}\right\|_{1}\|w\|_{\ell^{\infty}}=\kappa\|w\|_{\ell^{\infty}} .
\end{aligned}
$$

In the last inequality, we have used Assumption 1. 


\section{References}

[1] R. Olfati-Saber, A. Fax and R.M. Murray, Consensus and cooperation in networked multi-agent systems, Proc. of IEEE, 95(1) (2007) 215-233.

[2] G.N. Nair, F. Fagnani, S. Zampieri and R.J. Evans, Feedback control under data rate constraints: an overview, Proc. of IEEE, 95(1) (2007) 108-137.

[3] S. Tatikonda and S.K. Mitter, Control under communication constraints, IEEE Trans. Automat. Contr., 49(7) (2004) 1056-1068.

[4] J.P. Hespanha, P. Naghshtabrizi and Y. Xu, A survey of recent results in networked control systems, Proc. of the IEEE, 95(1) (2007) 138-162.

[5] H. Ishii, $H^{\infty}$ control with limited communication and message losses, Syst. Contr. Lett., 57 (2008) 322-331.

[6] S. Hu and W.-Y. Yan, Stability analysis of networked control systems with respect to packet loss, Automatica, 43(7) (2007) 1243-1248.

[7] L. Hetel, J. Daafouz and C. Iung, Stabilization of arbitrary switched linear systems with unknown time-varying delays, IEEE Trans. Automat. Contr., 51(10) (2006) 1668-1674.

[8] Y.-J. Pan, H.J. Marquez and T. Chen, Stabilization of remote control systems with unknown time varying delays by LMI techniques, Int. Journal of Control, 79(7) (2006) 752-763.

[9] K. Tsumura, H. Ishii and H. Hoshina, Tradeoffs between quantization and packet loss in networked control of linear systems, Automatica, 45(12) (2009) 2963-2970.

[10] Y. Niu, T. Jia, X. Wang and F. Yang, Output-feedback control design for NCSs subject to quantization and dropout, Information Sciences, 179 (2009) 3804-3813.

[11] N. Elia and S.K. Mitter, Stabilization of linear systems with limited information, IEEE Trans. Automat. Contr., 45 (2001) 1279-1289. 
[12] A.S. Matveev and A.V. Savkin, Comments on "Control over noisy channels" and relevant negative results, IEEE Trans. Automat. Contr., 50(12) (2005) 2105-2110.

[13] N.C. Martins, Finite gain $l_{p}$ stabilization requires analog control, Syst. Contr. Lett., 55 (2006) 949-954.

[14] E.D. Sontag, Smooth stabilization implies coprime factorization, IEEE Trans. Automat. Contr., 34(4) (1989) 435-443.

[15] D.E. Quevedo, E.I. Silva and G.C. Goodwin, Packetized predictive control over erasure channels, Proc. of American Control Conference (2007)

[16] D.E. Quevedo and D. Nešić, Input-to-state stability of packetized predictive control over unreliable networks affected by packet-dropouts, IEEE Trans. Automat. Contr., 56(3) (2011) 370-375.

[17] A. Bemporad, Predictive control of teleoperated constrained systems with unbounded communication delays, Proc. IEEE Conf. Decis. Contr., (1998) 2133-2138.

[18] P.L. Tang and C.W. de Silva, Stability validation of a constrained model predictive networked control system with future input buffering, Int. J. Contr., 80(12) (2007) 1954-1970.

[19] Y. Ishido and K. Takaba, Robust $\ell^{p}$ stability and robust $\ell^{\infty}$ stabilization over a rate-limited digital channel, Proc. of 1st IFAC Workshop on Distributed Estimation and Control in Networked Systems (NecSys'09), Venice, Italy (2009) 298-303.

[20] Y. Ishido and K. Takaba, Robust $\ell^{p}$ stability and robust $\ell^{\infty}$ stabilization over a rate-limited digital channel, SICE Journal of Control, Measurement, and System Integration, 3(2) (2010) 101-110.

[21] Y. Ishido and K. Takaba, A new framework for robust stability analysis of quantized feedback systems, Proc. of 2010 American Control Conference (2010), 3901-3906.

[22] H.K. Khalil, Nonlinear Systems 3rd Edition, Prentice Hall (2002)

[23] H. Bourles, Local $\ell^{p}$-stability and local small gain theorem for discretetime systems, IEEE Trans. Automat. Contr., 41(6) (1996) 903-907. 